
053620170108

\title{
Assessment of resistances to multiple pathogens in experimental sweet pepper hybrids
}

\author{
Regis C Carvalho ${ }^{1}$; Douglas W Nogueira ${ }^{1}$; César A Ticona-Benavente ${ }^{2}$; Danilo G Nogueira ${ }^{1}$; Wilson R \\ Maluf $^{1}$; Ranoel JS Gonçalves ${ }^{3}$; Luis FL Silva ${ }^{1}$ \\ 'Universidade Federal de Lavras (UFLA), Lavras-MG, Brasil; regisccarvalho@hotmail.com; wrmaluf@dag.ufla.br; douglagen@yahoo. \\ com.br; asp.nogueira@yahoo.com.br; luisufla@hotmail.com; ${ }^{2}$ Instituto Nacional de Pesquisas da Amazônia (INPA), Manaus-AM, Brasil; \\ cesar.benavente@gmail.com;3'Universidade Federal de Campina Grande (UFCG), Sume-PB, Brasil; ranoelgoncalves@hotmail.com
}

\begin{abstract}
The aim of this study was to assess resistance to some of the major sweet pepper pathogens [Phytophthora capsici, Pepper yellow mosaic virus (PepYMV) and Meloidogyne incognita] in a group of experimental hybrids and their respective parental lines, and to identify hybrids possibly resistant to all three pathogens. Ten parental breeding lines, thirty experimental hybrids and seven commercial controls (Konan-R, Magali-R, Martha-R, Stephany, Mallorca, Magnata Super and Criollo de Morelos-334) were used. Each experiment was set up in a randomized complete block design with three replications and plots consisting of 16 plants. For assessment of resistance to $P$. capsici and PepYMV, the percent of asymptomatic plants was considered. In evaluating reactions to $M$. incognita, both the nematode reproduction index and the nematode reproduction factor were calculated. Five hybrids were found with resistance to all three pathogens. For all three pathogens, there was evidence that hybrids with two resistant parental lines showed slightly higher levels of pathogen resistance than those with only one resistant parental line.
\end{abstract}

Keywords: Capsicum annuum, Pepper yellow mosaic virus, Phytophthora capsici, Meloidogyne incognita.

\section{RESUMO}

Caracterização das resistências a múltiplos patógenos em híbridos experimentais de pimentão

O objetivo deste trabalho foi caracterizar híbridos experimentais de pimentão e suas linhagens parentais, quanto às reações de resistência a Phytophthora capsici, Pepper yellow mosaic virus (PepYMV) e Meloidogyne incognita, e identificar híbridos possivelmente resistentes a esses três patógenos. Foram utilizadas 10 linhagens, 30 híbridos experimentais, e sete testemunhas comerciais (Konan-R, Magali-R, Martha-R, Stephany, Mallorca, Magnata Super e Criollo de Morelos-334). Para cada um dos três experimentos, utilizou-se o delineamento em blocos casualizados, com três repetições. $\mathrm{Na}$ avaliação das reações a $P$. capsici e ao PepYMV, foram consideradas as percentagens de plantas sem sintomas. $\mathrm{Na}$ avaliação das reações a $M$. incognita, foram calculados o índice de reprodução e o fator de reprodução do nematoide. Foram identificados cinco híbridos com resistência aos três patógenos. Para os três patógenos foram encontradas evidências de que híbridos com duas linhagens parentais resistentes apresentam níveis de resistência ligeiramente superiores àqueles com apenas uma linhagem parental resistente.

Palavras-chave: Capsicum annuum, Pepper yellow mosaic virus, Phytophthora capsici, Meloidogyne incognita.

\section{Received on September 14, 2015; accepted on July 22, 2016}

$\mathrm{T}$ here has been a significant increase in sweet pepper production in Brazil in recent years (Nogueira, 2010). The most highly used cultivars are $\mathrm{F}_{1}$ hybrids. The main reasons for growing use of these hybrids are because of: (a) heterosis for yield in sweet peppers (Nascimento et al., 2004, 2010; Gomide et al., 2008), and (b) greater resistance of hybrids to diseases as a result of the dominant nature of the genes that control these resistances, a fact which favors acquisition of multiple resistances through being able to more easily associate, in a single hybrid, the resistances normally found in separate parents.

One of the main problems of growing sweet peppers in Brazil is the occurrence of diseases. The fungus Phytophthora capsici, the potyvirus and the nematode Meloidogyne incognita stand out as pathogens of importance. $P$. capsici is the causal agent of the disease known as wilt, blight or root rot, one of the most destructive diseases of this crop throughout the world (McGregor et al., 2011). Among the potyvirus, PepYMV (Pepper yellow mosaic virus) is of greatest prevalence in the country (Lucinda et al., 2012), causing yellow mosaic virus in sweet peppers. Meloidogyne incognita may lead to the greatest damage in sweet pepper because it occurs most frequently in crop fields (Lopes \& Ávila, 2003).

Genetic resistance to these plant pathogens is one of the most efficient ways of minimizing damages, as well as having less impact on the environment. The existence of genetic variability in the Capsicum genus has 
allowed the introduction of resistance to these pathogens in sweet pepper cultivars (Candole et al., 2010; Fazari et al., 2012; Nogueira et al., 2012). In general, genetic resistance to these plant pathogens may be exploited in hybrid combinations because studies show that genetic control of resistance is usually due to the presence of dominant alleles, with monogenic inheritance (Echer \& Costa, 2002; Monroy-Barbosa \& Bosland, 2008; Thies \& Ariss, 2009). Currently, sweet pepper cultivars in Brazil show resistance to only one or two of the pathogens. Hybrids resistant to potyvirus are more common; less frequent are those with double resistance (to both potyvirus) and to $P$. capsici. Cultivars with resistance to $M$. incognita are practically non-existent.

The aim of this study was to assess experimental sweet pepper hybrids and their parental lines in regard to reactions of resistance to Phytophthora capsici, Pepper yellow mosaic virus (PepYMV) and Meloidogyne incognita; and identify hybrids possibly resistant to all three pathogens.

\section{MATERIAL AND METHODS}

Genetic material consisted of 47 sweet pepper genotypes: 10 lines (used as parents in obtaining experimental hybrids), 30 experimental hybrids, and seven commercial controls (Konan-R, Magali-R, Martha-R, Stephany, Mallorca, Magnata Super and Criollo de Morelos-334). The control Criollo de Morelos-334 was resistant to the three diseases, and Magnata Super was susceptible to the three. The parental lines and their presumed characteristics are described in Table 1. The lines with a PIX code were derived from breeding programs conducted in the company HortiAgro Sementes and are presumably resistant to PepYMV and to $P$. capsici and susceptible to $M$. incognita. PIM-013 is an elite line from HortiAgro, with resistance to $P$. capsici and susceptibility to both PepYMV and to M. incognita. Myr-29-09-05 and Myr29-11-08 were HortiAgro lines selected for greater uniformity in fruit shape from the Myr-29 open pollination population, considered to be resistant to PepYMV. Myr-29-09-05 and Myr-29-11-08 are resistant to PepYMV, but a priori are considered to be susceptible to both $P$. capsici and to $M$. incognita. Carolina Wonder and Charleston Belle are lines obtained from the U.S. Vegetable Laboratory, USDA/ARS, Charleston, SC, USA. They are homozygous for the $N$ gene, which confers resistance to the nematode $M$. incognita (Fery et al., 1998); and they probably have reactions of susceptibility to PepYMV and to $P$. capsici.

Reactions of the sweet pepper genotypes to Phytophthora capsici, PepYMV and Meloidogyne incognita were assessed in three separate experiments in a protected greenhouse at the Experimental Station of HortiAgro Sementes S.A. on the Palmital Farm in the municipality of Ijaci-MG, Brazil (21 ${ }^{\circ} 14^{\prime} 16^{\prime} \mathrm{S}, 45^{\circ} 08^{\prime} 00^{\prime \prime} \mathrm{W}$, altitude of $920 \mathrm{~m}$ ) in March 2012.

In each experiment, the treatments were sown in 128-cell Styrofoam trays (16x8) containing commercial substrate. A randomized block experimental design was used, with three replications and sixteen plants per experimental unit. A treatment was added only for the experiment with $M$. incognita, a susceptible line of tomato TOM-584, for the purpose of calculating the reproduction index and factor.

In assessment of reactions to Phytophthora capsici, the isolates Pc11 and Pc31 of the pathogen, granted by the company Sakata Sudamerica/Agroflora, Bragança Paulista-SP, Brazil (originally collected in the region of Bernardino de Campos-SP and Santa Cruz do Rio Pardo-SP, respectively), were kept in test tubes containing PDA medium (Potato Dextrose Agar) and stored in a BOD (Biochemical Oxygen Demand) chamber. Maintenance and production of the inoculum were carried out according to Nascimento et al. (2007).

Inoculations were carried out on seedlings kept in Styrofoam trays, obtained as described above. An inoculum concentration of $10^{4}$ zoospores/mL was used, applying $5 \mathrm{~mL}$ of the suspension in each tray cell, near the root collar of the plants at 40 days after germination. After inoculation, assessments were made as of the third day after inoculation, extending to the $15^{\text {th }}$ day. Scores were attributed for each plant ranging from 1 to 3 , with $1=$ Healthy, 2= Necrosis and wilting; $3=$ Leafless and dry. The plants that had a score of 1 in assessment at day 15 were considered as being asymptomatic.

In the experiment of assessment of reactions to PepYMV, a potyvirus isolate was used, serologically designated as PepYMV, granted by the company Sakata Sudamerica and obtained in the region of Lins-SP, derived from sweet pepper plants with systemic symptoms and naturally infected. To maintain the isolate, Turkish "TNN" tobacco plants and sweet peppers of the Ikeda cultivar (susceptible to PepYMV), previously infected with PepYMV, were stored in desiccators with silica-gel and also in liquid nitrogen in an ultra-freezer at $-80^{\circ} \mathrm{C}$. For production of the inoculum, multiplication was carried out in tobacco plants of the cultivar TNN, kept in screened greenhouses and replaced at intervals no greater than two months.

For inoculation in sweet peppers, tobacco leaves infected with PepYMV, used as a source of inoculum, were macerated in a $0.01 \mathrm{M}$ phosphate buffer, $\mathrm{pH}$ 7.0. Then the sweet pepper plants to be tested were sprinkled with silicon carbide (400 mesh) and, afterwards, the plant extract solution was applied by rubbing the thumb on the leaves. After inoculation, the plants were irrigated and kept in greenhouses with plastic covering and screened sides. Two inoculations were carried out to avoid possible escapes; the first when the plants reached the first fully-expanded definitive leaf stage, and the second at seven days after the first.

Assessments were carried out weekly, from the $15^{\text {th }}$ to the $40^{\text {th }}$ day after the first inoculation, for a total of five assessments; definitive assessments were taken as those made on the $40^{\text {th }}$ day. Scores were attributed to each plant ranging from 1 to 5 , with $1=$ no symptoms; $2=1$ loss of color in the intervein area; $3=$ light mosaic; $4=$ well developed mosaic, without leaf deformation; 5= yellow mosaic, blistered, with leaf deformation. Plants that had a score of 1 on the assessment 
of the $40^{\text {th }}$ day were considered to be asymptomatic.

In assessments of reactions to $M$. incognita, the reproduction index and the reproduction factor of the nematode were calculated. The isolate known as $M$. incognita was used as a source of inoculum, which was previously multiplied and kept on the susceptible tomato Solanum lycopersicum, Santa Clara cultivar. The eggs of the nematodes were extracted following the method of Bonetti \& Ferraz (1981). The roots containing galls of the tomato plant were cut in pieces of approximately $0.5 \mathrm{~cm}$ length and ground up in a blender for 40 seconds with a sodium hypochloride solution at $0.5 \%$. Then, the solution containing the eggs was poured into a sieve with mesh openings of $0.074 \mathrm{~mm}$, over a sieve with a mesh of $0.028 \mathrm{~mm}$. The eggs were subjected to complete washing under running water.

The nematode eggs, retained in the sieve with the smaller mesh, were collected and quantified in a stereo microscope. The plants were inoculated 15 days after germination using an automatic syringe for veterinary use. For inoculation of each seedling in the trays, an aliquot of solution containing 1208 viable eggs was used, quantified by eclosion chambers.

Assessments were made at 60 days after inoculation, following the method of Bonetti \& Ferraz (1981). The number of eggs/gram of root was calculated by dividing the number of eggs by the fresh weight of the root. The value of the reproduction index was calculated as follows: [number of eggs per gram of root of each experimental unit (plot)/ mean number of eggs per gram of root of the tomato plants TOM-584] x 100 . The value of the reproduction factor was calculated as follows: final population/ initial population of viable eggs.

The data were subjected to analysis of variance and, when significant, the mean values were compared by the Tukey test and Dunnett test at 5\% probability.

\section{RESULTS AND DISCUSSION}

Except for the lines PIX-044B-01-01 (for PepYMV), Carolina Wonder and Charleston Belle (for P. capsici), and Myr-29-09-05 (for M. incognita), all the others exhibited the expected or presumed reactions in relation to $P$. capsici, PepYMV and M.incognita (Tables 2 and 3).

PIX-044B-01-01, PIX-044B-13-01, PIX-045B-27-02, PIX-045B-32-03, PIX-052B-06-01 and PIM-013 did not differ from the resistant control Criollo de Morelos-334 in regard to reaction to $P$. capsici, but they differed significantly in regard to the susceptible control Magnata Super, and were thus considered resistant to the pathogen (Table 2). Carolina Wonder and Charleston Belle proved to have intermediate resistance to $P$. capsici (27.1 and $35.4 \%$ asymptomatic plants), differing from both the resistant control and the susceptible control by the Dunnett test. The moderate resistance of Carolina Wonder and Charleston Belle to $P$. capsici was not expected from the description made by the authors (Fery et al., 1998), and may presumably be explained by the presence of one or more genes that confer this resistance. The other lines showed susceptibility to P. capsici, not differing from Magnata Super; the percentage of asymptomatic plants was near zero.

Inoculations with PepYMV showed that the lines PIX-044B-13-01, PIX045B-27-02, PIX-045B-32-03, PIX-
052B-06-01, Myr-29-09-05 and Myr29-11-8 may be considered resistant to the pathogen since they showed percentages of plants without mosaic symptoms at or near $100 \%$, not differing from the resistant control Criollo de Morellos-334 (Table 2). The lines presumably susceptible to PepYMV (Carolina Wonder, Charleston Belle, PIM-013) did not show asymptomatic plants and did not differ from the susceptible control Magnata Super, and their susceptibility was thus confirmed. Only the line PIX-044B-01-01 showed intermediate values $(78.3 \%$ resistant plants), differing from both the resistant control and the susceptible control, in contrast with the initially presumed reaction of resistance. Since the trait is of monogenic inheritance (Echer \& Costa, 2002), and PIX-044B-01-01 corresponds to an $\mathrm{F}_{4}$ family, an explanation for this result would be the fact that the line constitutes a segregating family for resistance to the virus.

Inoculations with $M$. incognita showed that three of the parental lines tested (Carolina Wonder, Charleston Belle and Myr-29-09-05) have high levels of resistance to the nematode, with reproduction indices (RI\%) and reproduction factors (RF) near zero, which did not differ from the resistant control Criollo de Morellos-334 (Table $3)$. All the other parental lines tested did not differ from those found in the

Table 1. Description of the parental lines of sweet pepper. Lavras, UFLA, 2012.

\begin{tabular}{lccc}
\hline Parental lines & \multicolumn{3}{c}{ Reaction $^{1}$} \\
\cline { 2 - 4 } & $\begin{array}{c}\text { Phytophthora } \\
\text { capsici }\end{array}$ & PepYMV & $\begin{array}{c}\text { Meloidogyne } \\
\text { incognita }\end{array}$ \\
\hline PIX-044B-01-01 & $\mathrm{R}(?)$ & $\mathrm{R}(?)$ & $\mathrm{S}(?)$ \\
PIX-044B-13-01 & $\mathrm{R}(?)$ & $\mathrm{R}(?)$ & $\mathrm{S}(?)$ \\
PIX-045B-27-02 & $\mathrm{R}(?)$ & $\mathrm{R}(?)$ & $\mathrm{S}(?)$ \\
PIX-045B-32-03 & $\mathrm{R}(?)$ & $\mathrm{R}(?)$ & $\mathrm{S}(?)$ \\
PIX-052B-06-01 & $\mathrm{R}(?)$ & $\mathrm{R}(?)$ & $\mathrm{S}(?)$ \\
CarolinaWonder & $\mathrm{S}(?)$ & $\mathrm{S}(?)$ & $\mathrm{R}$ \\
Charleston Belle & $\mathrm{S}(?)$ & $\mathrm{S}(?)$ & $\mathrm{R}$ \\
MYR-29-09-05 & $\mathrm{S}(?)$ & $\mathrm{R}$ & $\mathrm{S}(?)$ \\
MYR-29-11-08 & $\mathrm{S}(?)$ & $\mathrm{R}$ & $\mathrm{S}(?)$ \\
PIM-13 & $\mathrm{R}$ & $\mathrm{S}$ & $\mathrm{S}$ \\
\hline
\end{tabular}

${ }^{1} \mathrm{~S}=$ susceptibility; $\mathrm{R}=$ resistance; $(?)=$ indicates presumed resistance $(\mathrm{R})$ or susceptibility $(\mathrm{S})$ based on genealogy and/or previously obtained information. They are subject to confirmation in the present trials. 
Table 2. Assessment of reaction of resistance to Phytophthora capsici and to PepYMV in sweet pepper. Lavras, UFLA, 2012.

\begin{tabular}{|c|c|c|c|c|c|c|c|}
\hline \multirow[b]{2}{*}{ Treatment } & \multicolumn{3}{|c|}{ Phytophthora capsici } & \multicolumn{4}{|c|}{ PepYMV } \\
\hline & $\begin{array}{c}\text { Plants without } \\
\text { symptoms }^{1} \\
(\%)\end{array}$ & $\begin{array}{c}\text { Dunnett } \\
\text { Pr }<\text { Criollo de } \\
\text { Morelos }^{2}\end{array}$ & $\begin{array}{c}\text { Dunnett } \\
\text { Pr>Magnata } \\
\text { Super }^{3}\end{array}$ & $\begin{array}{r}\text { Plant } \\
\text { sym }\end{array}$ & $\begin{array}{l}\text { S without } \\
\text { iptoms }{ }^{1} \\
(\%)\end{array}$ & $\begin{array}{c}\text { Dunnett } \\
\text { Pr }<\text { Criollo } \\
\text { de } \text { Morelos }^{2}\end{array}$ & $\begin{array}{c}\text { Dunnett } \\
\text { Pr }>\text { Magnata } \text { Super }^{3}\end{array}$ \\
\hline PIX-044B-01-01 & $100 \mathrm{a}$ & ns & $* *$ & 78.3 & bcd & $* *$ & $* *$ \\
\hline PIX-044B-13-01 & $100 \mathrm{a}$ & ns & $* *$ & 100 & $\mathrm{a}$ & ns & $* *$ \\
\hline PIX-045B-27-02 & $93.9 \mathrm{ab}$ & ns & $* *$ & 100 & $\mathrm{a}$ & ns & $* *$ \\
\hline PIX-045B-32-03 & $100 \mathrm{a}$ & ns & $* *$ & 100 & $\mathrm{a}$ & ns & $* *$ \\
\hline PIX-052B-06-01 & $100 \mathrm{a}$ & $\mathrm{ns}$ & $* *$ & 93 & $\mathrm{abc}$ & ns & $* *$ \\
\hline Carolina Wonder & $27.1 \mathrm{ef}$ & $* *$ & $* *$ & 0 & e & $* *$ & ns \\
\hline Charleston Belle & $35.4 \mathrm{e}$ & $* *$ & $* *$ & 0 & e & $* *$ & ns \\
\hline MYR-29-09-05 & $0 \mathrm{~g}$ & $* *$ & $\mathrm{~ns}$ & 100 & $\mathrm{a}$ & ns & $* *$ \\
\hline MYR-29-11-08 & $0 \mathrm{~g}$ & $* *$ & $\mathrm{~ns}$ & 100 & $\mathrm{a}$ & ns & $* *$ \\
\hline PIM-013 & $100 \mathrm{a}$ & ns & $* *$ & 0 & e & $* *$ & ns \\
\hline Konan-R & $100 \mathrm{a}$ & ns & $* *$ & 100 & $\mathrm{a}$ & ns & $* *$ \\
\hline Magali-R & $0 \mathrm{~g}$ & $* *$ & ns & 100 & $\mathrm{a}$ & ns & $* *$ \\
\hline Martha-R & $100 \mathrm{a}$ & ns & $* *$ & 100 & $\mathrm{a}$ & ns & $* *$ \\
\hline Stephany & $91.4 \mathrm{abc}$ & ns & $* *$ & 97.4 & $\mathrm{ab}$ & ns & $* *$ \\
\hline Mallorca & 97.9 a & ns & $* *$ & 100 & $\mathrm{a}$ & ns & $* *$ \\
\hline Magnata Super & $2.1 \mathrm{fg}$ & $* *$ & & 0 & $\mathrm{e}$ & $* *$ & \\
\hline Criollo de Morelos-334 & $100 \mathrm{a}$ & & $* *$ & 100 & $\mathrm{a}$ & & $* *$ \\
\hline $\begin{array}{l}\text { F1(PIX-044B-01-01 X } \\
\text { Carolina Wonder) }\end{array}$ & $93.8 \mathrm{ab}$ & ns & $* *$ & 88.1 & $a b c$ & ns & $* *$ \\
\hline $\begin{array}{l}\text { F1(PIX-044B-13-01 X } \\
\text { Carolina Wonder) }\end{array}$ & 85.4 abcd & ns & $* *$ & 100 & $\mathrm{a}$ & ns & $* *$ \\
\hline $\begin{array}{l}\text { F1(PIX-045B-27-02 X } \\
\text { Carolina Wonder) }\end{array}$ & $81.3 \mathrm{abcd}$ & $*$ & $* *$ & 100 & $\mathrm{a}$ & ns & $* *$ \\
\hline $\begin{array}{l}\text { F1(PIX-045B-32-03 X } \\
\text { Carolina Wonder) }\end{array}$ & $100 \mathrm{a}$ & ns & $* *$ & 74.9 & $\mathrm{~cd}$ & $* *$ & $* *$ \\
\hline $\begin{array}{l}\text { F1(PIX-052B-06-01 X } \\
\text { Carolina Wonder) }\end{array}$ & $77.1 \mathrm{abcd}$ & $* *$ & $* *$ & 80.6 & abcd & $* *$ & $* *$ \\
\hline $\begin{array}{l}\text { F1(PIX-044B-01-01 X } \\
\text { Charleston Belle) }\end{array}$ & $93.8 \mathrm{ab}$ & ns & $* *$ & 61.9 & $\mathrm{~d}$ & $* *$ & $* *$ \\
\hline $\begin{array}{l}\text { F1(PIX-044B-13-01 X } \\
\text { Charleston Belle) }\end{array}$ & $64.6 \mathrm{~d}$ & $* *$ & $* *$ & 100 & $\mathrm{a}$ & ns & $* *$ \\
\hline $\begin{array}{l}\text { F1(PIX-045B-27-02 X } \\
\text { Charleston Belle) }\end{array}$ & $77.1 \mathrm{abcd}$ & $* *$ & $* *$ & 97.8 & $\mathrm{ab}$ & ns & $* *$ \\
\hline $\begin{array}{l}\text { F1(PIX-045B-32-03 X } \\
\text { Charleston Belle) }\end{array}$ & $97.9 \mathrm{a}$ & $\mathrm{ns}$ & $* *$ & 77.5 & $\mathrm{~cd}$ & $* *$ & $* *$ \\
\hline $\begin{array}{l}\text { F1(PIX-052B-06-01 X } \\
\text { Charleston Belle) }\end{array}$ & $93.8 \mathrm{ab}$ & ns & $* *$ & 78.3 & bcd & $* *$ & $* *$ \\
\hline $\begin{array}{l}\text { F1(Carolina Wonder X } \\
\text { MYR-29-09-05) }\end{array}$ & $33.3 \mathrm{e}$ & $* *$ & $* *$ & 100 & $\mathrm{a}$ & ns & $* *$ \\
\hline $\begin{array}{l}\text { F1(Charleston Belle X } \\
\text { MYR-29-09-05) }\end{array}$ & $27.1 \mathrm{ef}$ & $* *$ & $* *$ & 100 & $\mathrm{a}$ & ns & $* *$ \\
\hline $\begin{array}{l}\text { F1(PIM-013 X MYR- } \\
\text { 29-09-05) }\end{array}$ & $100 \mathrm{a}$ & $\mathrm{ns}$ & $* *$ & 100 & $\mathrm{a}$ & ns & $* *$ \\
\hline
\end{tabular}




\begin{tabular}{|c|c|c|c|c|c|c|c|}
\hline \multirow[b]{2}{*}{ Treatment } & \multicolumn{3}{|c|}{ Phytophthora capsici } & \multicolumn{4}{|c|}{ PepYMV } \\
\hline & $\begin{array}{c}\text { Plants without } \\
\text { symptoms }^{1} \\
(\%)\end{array}$ & $\begin{array}{c}\text { Dunnett } \\
\text { Pr }<\text { Criollo de } \\
\text { Morelos }^{2}\end{array}$ & $\begin{array}{c}\text { Dunnett } \\
\text { Pr }>\text { Magnata } \\
\text { Super }^{3}\end{array}$ & $\begin{array}{r}\text { Plant } \\
\text { sym }\end{array}$ & $\begin{array}{l}\text { S without } \\
\text { iptoms } \\
(\%) \\
(\%)\end{array}$ & $\begin{array}{c}\text { Dunnett } \\
\text { Pr }<\text { Criollo } \\
\text { de } \text { Morelos }^{2}\end{array}$ & $\begin{array}{c}\text { Dunnett } \\
\text { Pr>Magnata } \\
\text { Super }^{3}\end{array}$ \\
\hline MYR-29-09-05) & 68.8 & $* *$ & $* *$ & 100 & $\mathrm{a}$ & ns & $* *$ \\
\hline $\begin{array}{l}\text { F1(PIX-044B-13-01 X } \\
\text { MYR-29-09-05) }\end{array}$ & $64.6 \mathrm{~d}$ & $* *$ & $* *$ & 100 & $\mathrm{a}$ & ns & $* *$ \\
\hline $\begin{array}{l}\text { F1(PIX-045B-27-02 X } \\
\text { MYR-29-09-05) }\end{array}$ & $66.7 \mathrm{~cd}$ & $* *$ & $* *$ & 100 & $\mathrm{a}$ & ns & $* *$ \\
\hline $\begin{array}{l}\text { F1(PIX-045B-32-03 X } \\
\text { MYR-29-09-05) }\end{array}$ & $95.8 \mathrm{a}$ & $\mathrm{ns}$ & $* *$ & 100 & $\mathrm{a}$ & $\mathrm{ns}$ & $* *$ \\
\hline $\begin{array}{l}\text { F1(PIX-052B-06-01 X } \\
\text { MYR-29-09-05) }\end{array}$ & 95.8 & ns & $* *$ & 100 & $\mathrm{a}$ & ns & $* *$ \\
\hline $\begin{array}{l}\text { F1(Carolina Wonder X } \\
\text { MYR-29-11-08) }\end{array}$ & $4.2 \mathrm{fg}$ & $* *$ & ns & 100 & $\mathrm{a}$ & ns & $* *$ \\
\hline $\begin{array}{l}\text { F1(Charleston Belle X } \\
\text { MYR-29-11-08) }\end{array}$ & $2.1 \mathrm{fg}$ & $* *$ & ns & 100 & $\mathrm{a}$ & ns & $* *$ \\
\hline $\begin{array}{l}\text { F1(PIM-013 X MYR- } \\
\text { 29-11-08) }\end{array}$ & $100 \mathrm{a}$ & ns & $* *$ & 100 & $\mathrm{a}$ & ns & $* *$ \\
\hline $\begin{array}{l}\text { F1(PIX-044B-01-01 X } \\
\text { MYR-29-11-08) }\end{array}$ & $95.8 \mathrm{a}$ & ns & $* *$ & 100 & $\mathrm{a}$ & ns & $* *$ \\
\hline $\begin{array}{l}\text { F1(PIX-044B-13-01 X } \\
\text { MYR-29-11-08) }\end{array}$ & $93.8 \mathrm{ab}$ & ns & $* *$ & 100 & $\mathrm{a}$ & ns & $* *$ \\
\hline $\begin{array}{l}\text { F1(PIX-045B-27-02 X } \\
\text { MYR-29-11-08) }\end{array}$ & $87.5 \mathrm{abcd}$ & ns & $* *$ & 100 & $\mathrm{a}$ & ns & $* *$ \\
\hline $\begin{array}{l}\text { F1(PIX-045B-32-03 X } \\
\text { MYR-29-11-08) }\end{array}$ & $95.8 \mathrm{a}$ & ns & $* *$ & 100 & $\mathrm{a}$ & ns & $* *$ \\
\hline $\begin{array}{l}\text { F1(PIX-052B-06-01 X } \\
\text { MYR-29-11-08) }\end{array}$ & $93.8 \mathrm{ab}$ & ns & $* *$ & 100 & $\mathrm{a}$ & ns & $* *$ \\
\hline $\begin{array}{l}\text { F1(PIX-044B-01-01 X } \\
\text { PIM-013) }\end{array}$ & $100 \mathrm{a}$ & ns & $* *$ & 86.3 & $\mathrm{abc}$ & ns & $* *$ \\
\hline $\begin{array}{l}\text { F1(PIX-044B-13-01 X } \\
\text { PIX-052B-06-01) }\end{array}$ & $100 \mathrm{a}$ & ns & $* *$ & 100 & $\mathrm{a}$ & ns & $* *$ \\
\hline $\begin{array}{l}\text { F1(PIX-045B-27-02 X } \\
\text { PIX-052B-06-01) }\end{array}$ & $100 \mathrm{a}$ & ns & $* *$ & 100 & $\mathrm{a}$ & ns & $* *$ \\
\hline $\begin{array}{l}\text { F1(PIX-045B-32-03 X } \\
\text { PIX-052B-06-01) }\end{array}$ & $100 \mathrm{a}$ & ns & $* *$ & 88.9 & $\mathrm{abc}$ & ns & $* *$ \\
\hline
\end{tabular}

${ }^{1}$ Mean values followed by the same letter in the column do not differ among themselves by Tukey test at 5\% probability. ${ }^{2}$ Control resistant to Phytophthora capsici and to PepYMV. ${ }^{3}$ Control susceptible to Phytophthora capsici and to PepYMV. ${ }^{\text {ns }}, * * * *=$ not significant, significant at $1 \%$ probability, and significant at $5 \%$ probability by the Dunnett test, respectively.

susceptible control Magnata Super. In the lines Carolina Wonder and Charleston Belle, these results were expected from the description of their authors (Fery et al., 1998), who report that they are homozygous for the $N$ allele that confers resistance to $M$. incognita. However, this information was not available for the line Myr-29-
09-05, which was presumed a priori to be susceptible since the open pollination cultivar that gave rise to it (Myr-29) is not considered to be resistant. It is possible that Myr-29 included a percentage of plants resistant to $M$. incognita, which would have allowed the selection of either a resistant line (Myr-29-09-05) or a susceptible line
(Myr-29-11-08).

Of the 30 experimental hybrids tested in regard to reaction to $P$. capsici, four [F1(PIX-044B-01-01 x PIM013), F1(PIX-044B-13-01 x PIX052B-06-01), F1(PIX-045B-27-02 x PIX-052B-06-01), F1(PIX-045B-32-03 $x$ PIX-052B-06-01)] had two parental lines confirmed as highly resistant 
Table 3. Reproduction index (RI\%) and reproduction factor (RF) of Meloidogyne incognita in sweet pepper. Lavras, UFLA, 2012.

\begin{tabular}{|c|c|c|c|c|c|c|c|c|}
\hline \multirow{3}{*}{$\begin{array}{l}\text { Treatments } \\
\text { PIX-044B-01-01 }\end{array}$} & \multicolumn{4}{|c|}{ Reproduction index } & \multicolumn{4}{|c|}{ Reproduction factor } \\
\hline & \multicolumn{2}{|c|}{$\operatorname{RI}(\%)^{1}$} & \multirow{2}{*}{$\begin{array}{c}\text { Dunnett } \\
\text { Pr>Criollo } \\
\text { de Morelos }{ }^{2} \\
* *\end{array}$} & \multirow{2}{*}{$\begin{array}{c}\begin{array}{c}\text { Dunnett } \\
\text { Pr }<\text { Magnata } \\
\text { Super }^{3}\end{array} \\
\mathrm{~ns}\end{array}$} & \multicolumn{2}{|c|}{$\mathbf{R F}^{1}$} & \multirow{2}{*}{$\begin{array}{c}\text { Dunnett } \\
\text { Pr>Criollo } \\
\text { de Morelos }{ }^{2} \\
* *\end{array}$} & \multirow{2}{*}{$\begin{array}{c}\text { Dunnett } \\
\text { Pr }<\text { Magnata }^{\text {Super }}{ }^{3} \\
\text { Supe }^{2} \\
\mathrm{~ns}\end{array}$} \\
\hline & 318 & de & & & 66.9 & $\mathrm{~cd}$ & & \\
\hline PIX-044B-13-01 & 197 & bcd & $* *$ & ns & 37.2 & abcd & $* *$ & ns \\
\hline PIX-045B-27-02 & 283 & cde & $* *$ & ns & 58.7 & $\mathrm{~cd}$ & $* *$ & ns \\
\hline PIX-045B-32-03 & 173 & bc & $* *$ & ns & 41.5 & $\mathrm{~cd}$ & $* *$ & ns \\
\hline PIX-052B-06-01 & 347 & $\mathrm{e}$ & $* *$ & ns & 58.2 & $\mathrm{~cd}$ & $* *$ & ns \\
\hline Carolina Wonder & 0.2 & $\mathrm{a}$ & ns & $* *$ & 0 & $\mathrm{a}$ & ns & $* *$ \\
\hline Charleston Belle & 2.7 & $\mathrm{a}$ & ns & $* *$ & 0.3 & $\mathrm{a}$ & ns & $* *$ \\
\hline MYR-29-09-05 & 1 & $\mathrm{a}$ & ns & $* *$ & 0.2 & $\mathrm{a}$ & ns & $* *$ \\
\hline MYR-29-11-08 & 280 & cde & $* *$ & ns & 57.6 & $\mathrm{~cd}$ & $* *$ & $\mathrm{~ns}$ \\
\hline PIM-013 & 159 & $\mathrm{bc}$ & $* *$ & ns & 34.7 & abcd & $* *$ & $\mathrm{~ns}$ \\
\hline Konan-R & 136 & $\mathrm{ab}$ & $* *$ & ns & 29.9 & $\mathrm{abc}$ & $*$ & ns \\
\hline Magali-R & 219 & bcde & $* *$ & ns & 49.6 & $\mathrm{~cd}$ & $* *$ & ns \\
\hline Martha-R & 233 & bcde & $* *$ & ns & 56.5 & $\mathrm{~cd}$ & $* *$ & ns \\
\hline Stephany & 269 & bcde & $* *$ & ns & 60.9 & $\mathrm{~cd}$ & $* *$ & ns \\
\hline Mallorca & 231 & bcde & $* *$ & ns & 54.5 & $\mathrm{~cd}$ & $* *$ & ns \\
\hline Magnata Super & 157 & bc & $* *$ & & 37.1 & abcd & $* *$ & \\
\hline Criollo de Morelos-334 & 1.6 & a & & $* *$ & 0.2 & $\mathrm{a}$ & & $* *$ \\
\hline $\begin{array}{l}\text { F1(PIX-044B-01-01 X } \\
\text { Carolina Wonder) }\end{array}$ & 2.1 & a & ns & $* *$ & 0.5 & $\mathrm{a}$ & ns & $* *$ \\
\hline $\begin{array}{l}\text { F1(PIX-044B-13-01 X } \\
\text { Carolina Wonder) }\end{array}$ & 0.1 & $\mathrm{a}$ & ns & $* *$ & 0 & $\mathrm{a}$ & ns & $* *$ \\
\hline $\begin{array}{l}\text { F1(PIX-045B-27-02 X } \\
\text { Carolina Wonder) }\end{array}$ & 1.9 & $\mathrm{a}$ & ns & $* *$ & 0.4 & $\mathrm{a}$ & ns & $* *$ \\
\hline $\begin{array}{l}\text { F1(PIX-045B-32-03 X } \\
\text { Carolina Wonder) }\end{array}$ & 6.2 & $\mathrm{a}$ & ns & $* *$ & 1.6 & $\mathrm{ab}$ & ns & $* *$ \\
\hline $\begin{array}{l}\text { F1(PIX-052B-06-01 X } \\
\text { Carolina Wonder) }\end{array}$ & 2.6 & $\mathrm{a}$ & ns & $* *$ & 0.6 & $\mathrm{a}$ & ns & $* *$ \\
\hline $\begin{array}{l}\text { F1(PIX-044B-01-01 X } \\
\text { Charleston Belle) }\end{array}$ & 1.5 & a & ns & $* *$ & 0.5 & $\mathrm{a}$ & ns & $* *$ \\
\hline $\begin{array}{l}\text { F1(PIX-044B-13-01 X } \\
\text { Charleston Belle) }\end{array}$ & 3.3 & $\mathrm{a}$ & ns & $* *$ & 0.7 & $\mathrm{a}$ & ns & $* *$ \\
\hline $\begin{array}{l}\text { F1(PIX-045B-27-02 X } \\
\text { Charleston Belle) }\end{array}$ & 0.8 & $\mathrm{a}$ & $\mathrm{ns}$ & $* *$ & 0.2 & $\mathrm{a}$ & ns & $* *$ \\
\hline $\begin{array}{l}\text { F1(PIX-045B-32-03 X } \\
\text { Charleston Belle) }\end{array}$ & 0.7 & $\mathrm{a}$ & $\mathrm{ns}$ & $* *$ & 0.2 & $\mathrm{a}$ & $\mathrm{ns}$ & $* *$ \\
\hline $\begin{array}{l}\text { F1(PIX-052B-06-01 X } \\
\text { Charleston Belle) }\end{array}$ & 0.9 & $\mathrm{a}$ & $\mathrm{ns}$ & $* *$ & 0.2 & $\mathrm{a}$ & ns & $* *$ \\
\hline $\begin{array}{l}\text { F1(Carolina Wonder X } \\
\text { MYR-29-09-05) }\end{array}$ & 0.2 & $\mathrm{a}$ & $\mathrm{ns}$ & $* *$ & 0 & $\mathrm{a}$ & ns & $* *$ \\
\hline $\begin{array}{l}\text { F1(Charleston Belle X } \\
\text { MYR-29-09-05) }\end{array}$ & 1.3 & $\mathrm{a}$ & $\mathrm{ns}$ & $* *$ & 0.3 & $\mathrm{a}$ & $\mathrm{ns}$ & $* *$ \\
\hline $\begin{array}{l}\text { F1(PIM-013 X MYR-29- } \\
\text { 09-05) }\end{array}$ & 1.3 & $\mathrm{a}$ & $\mathrm{ns}$ & $* *$ & 0.3 & $\mathrm{a}$ & ns & $* *$ \\
\hline
\end{tabular}




\begin{tabular}{|c|c|c|c|c|c|c|c|}
\hline \multirow[b]{2}{*}{ Treatments } & \multicolumn{3}{|c|}{ Reproduction index } & \multicolumn{4}{|c|}{ Reproduction factor } \\
\hline & $\operatorname{RI}(\%)^{1}$ & $\begin{array}{c}\text { Dunnett } \\
\text { Pr }>\text { Criollo de } \\
\text { Morelos }^{2} \\
\end{array}$ & $\begin{array}{c}\text { Dunnett } \\
\text { Pr }<\text { Magnata } \\
\text { Super }^{3} \\
\end{array}$ & RI & $\mathbf{F}^{1}$ & $\begin{array}{c}\text { Dunnett } \\
\text { Pr }>\text { Criollo } \\
\text { de Morelos } \\
\end{array}$ & $\begin{array}{c}\text { Dunnett } \\
\text { Pr }<\text { Magnata } \text { Super }^{3} \\
\end{array}$ \\
\hline $\begin{array}{l}\text { F1(PIX-044B-01-01 X } \\
\text { MYR-29-09-05) }\end{array}$ & $4.1 \mathrm{a}$ & ns & $* *$ & 1 & $\mathrm{a}$ & ns & $* *$ \\
\hline $\begin{array}{l}\text { F1(PIX-044B-13-01 X } \\
\text { MYR-29-09-05) }\end{array}$ & 0.9 a & ns & $* *$ & 0.2 & $\mathrm{a}$ & ns & $* *$ \\
\hline $\begin{array}{l}\text { F1(PIX-045B-27-02 X } \\
\text { MYR-29-09-05) }\end{array}$ & 5.8 a & ns & $* *$ & 1.8 & $\mathrm{ab}$ & ns & $* *$ \\
\hline $\begin{array}{l}\text { F1(PIX-045B-32-03 X } \\
\text { MYR-29-09-05) }\end{array}$ & $2.9 \mathrm{a}$ & ns & $* *$ & 0.7 & $\mathrm{a}$ & $\mathrm{ns}$ & $* *$ \\
\hline $\begin{array}{l}\text { F1(PIX-052B-06-01 X } \\
\text { MYR-29-09-05) }\end{array}$ & $4 \mathrm{a}$ & ns & $* *$ & 1 & $\mathrm{a}$ & ns & $* *$ \\
\hline $\begin{array}{l}\text { F1(Carolina Wonder X } \\
\text { MYR-29-11-08) }\end{array}$ & $2.4 \mathrm{a}$ & ns & $* *$ & 0.5 & $\mathrm{a}$ & ns & $* *$ \\
\hline $\begin{array}{l}\text { F1(Charleston Belle X } \\
\text { MYR-29-11-08) }\end{array}$ & $4.9 \mathrm{a}$ & ns & $* *$ & 1 & $\mathrm{a}$ & $\mathrm{ns}$ & $* *$ \\
\hline $\begin{array}{l}\text { F1(PIM-013 X MYR- } \\
\text { 29-11-08) }\end{array}$ & 252 bcde & $* *$ & ns & 65.9 & $\mathrm{~cd}$ & $* *$ & ns \\
\hline $\begin{array}{l}\text { F1(PIX-044B-01-01 X } \\
\text { MYR-29-11-08) }\end{array}$ & $190 \mathrm{bcd}$ & $* *$ & $\mathrm{~ns}$ & 45.7 & cd & $* *$ & ns \\
\hline $\begin{array}{l}\text { F1(PIX-044B-13-01 X } \\
\text { MYR-29-11-08) }\end{array}$ & $173 \mathrm{bc}$ & $* *$ & ns & 41.3 & $\mathrm{~cd}$ & $* *$ & $\mathrm{~ns}$ \\
\hline $\begin{array}{l}\text { F1(PIX-045B-27-02 X } \\
\text { MYR-29-11-08) }\end{array}$ & 283 cde & $* *$ & ns & 72.9 & $\mathrm{~d}$ & $* *$ & ns \\
\hline $\begin{array}{l}\text { F1(PIX-045B-32-03 X } \\
\text { MYR-29-11-08) }\end{array}$ & $179 \mathrm{bcd}$ & $* *$ & ns & 46.9 & cd & $* *$ & ns \\
\hline $\begin{array}{l}\text { F1(PIX-052B-06-01 X } \\
\text { MYR-29-11-08) }\end{array}$ & 233 bcde & $* *$ & ns & 63.9 & $\mathrm{~cd}$ & $* *$ & ns \\
\hline $\begin{array}{l}\text { F1(PIX-044B-01-01 X } \\
\text { PIM-013) }\end{array}$ & 241 bcde & $* *$ & ns & 68.2 & cd & $* *$ & ns \\
\hline $\begin{array}{l}\text { F1(PIX-044B-13-01 X } \\
\text { PIX-052B-06-01) }\end{array}$ & $155 \mathrm{bc}$ & $* *$ & $\mathrm{~ns}$ & 39.9 & bcd & $* *$ & ns \\
\hline $\begin{array}{l}\text { F1(PIX-045B-27-02 X } \\
\text { PIX-052B-06-01) }\end{array}$ & 238 bcde & $* *$ & ns & 57.8 & $\mathrm{~cd}$ & $* *$ & ns \\
\hline $\begin{array}{l}\text { F1(PIX-045B-32-03 X } \\
\text { PIX-052B-06-01) }\end{array}$ & $173 \mathrm{bc}$ & $* *$ & $\mathrm{~ns}$ & 51.9 & $\mathrm{~cd}$ & $* *$ & ns \\
\hline
\end{tabular}

${ }^{1}$ Mean values followed by the same letter in the column do not differ among themselves by Tukey test at $5 \%$ probability. ${ }^{2}$ Control resistant to Meloidogyne incognita. ${ }^{3}$ Control susceptible to Meloidogyne incognita. ${ }^{\text {ns }}, * * *=$ not significant, significant at $1 \%$ probability, and significant at $5 \%$ probability by the Dunnett test, respectively.

to $P$. capsici in their composition (Table 2). Such hybrids showed $100 \%$ asymptomatic plants after inoculation, a level comparable to those of the per se resistant lines, to that of the resistant control Criollo de Morelos-334, and to those of the resistant commercial hybrids Konan-R, Marta-R, Stephany and Mallorca. In contrast, 22 hybrids that had only one parent highly resistant to $P$. capsici in their constitution showed from $64.6 \%$ to $100 \%$ asymptomatic plants, always differing significantly from the susceptible control Magnata Super, but in some cases coming to be significantly less than those presented by the resistant control Criollo de Morellos-334. It is clear that the mean level of resistance of these hybrids is slightly less than that obtained in hybrids in which both parents are resistant. The allelic relations between the genes that control resistance to $P$. capsici in the resistant lines PIX-044B-01-01, PIX-044B-13-01, PIX-045B-27-02, PIX-045B-32-03, PIX-052B-06-01 and PIM-013 are not known, nor is it known if inheritance of the trait is monogenic. If the resistances in these cases are controlled by the same gene, it would 
be concluded that the homozygote would be slightly more resistant than the heterozygote, indicating incomplete dominance of the resistance allele. However, even if this allelism is not confirmed, an allelic dosage effect is clear since two resistant parents produce more resistant hybrids than those in which only one parent has resistance. Similar results were found for some genotypes evaluated in a study of Nascimento et al. (2007).

Four of the experimental hybrids tested [F1(Carolina Wonder x MYR29-09-05), F1(Charleston Belle x MYR-29-09-05), F1(Carolina Wonder x MYR-29-11-08), F1(Charleston Belle $x$ MYR-29-11-08)] had as a parent, on the one hand, a line with an intermediate level of resistance to $P$. capsici (Carolina Wonder or Charleston Belle), and, on the other hand, a susceptible line (Table 2). Among these four hybrids, the lowest levels of resistance to $P$. capsici, similar to those of the susceptible control Magnata Super, were associated with the F1(Carolina Wonder x MYR-2911-08) and F1(Charleston Belle $x$ MYR-29-11-08), in which one of the parents (Carolina Wonder or Charleston Belle) is resistant to the nematode $M$. incognita, and the other (MYR-2911-08) is susceptible. But the hybrids F1(Carolina Wonder x MYR-29-09-05) and F1(Charleston Belle x MYR-2909-05), in which both the parents are resistant to $M$. incognita, exhibited significantly greater levels of resistance to $P$. capsici than those of the control Magnata Super, comparable to those of the lines Carolina Wonder or Charleston Belle. A possible connection of the genes that control moderate resistance to $P$. capsici and the $N$ gene that controls resistance to $M$. incognita in Carolina Wonder/Charleston Belle could be a plausible explanation for these data if not for the fact that MYR-29-09-05, resistant to $M$. incognita, does not have even a moderate level of resistance to P. capsici.

None of the 30 experimental hybrids tested in regard to reaction to PepYMV were composed of two susceptible parents such that, in all cases, the percentages of asymptomatic plants ranged from 61.9 to $100 \%$, significantly higher than the values of $0 \%$ found in the susceptible genotypes Carolina Wonder, Charleston Belle, PIM-013 and Magnata Super (Table 2).

Eleven of the experimental hybrids tested [F1(PIX-044B-13-01 x MYR29-11-08), F1(PIX-045B-27-02 x MYR-29-11-08), F1(PIX-045B-32-03 X MYR-29-11-08), F1(PIX-052B-06-01 x MYR-29-11-08), F1(PIX-044B-13-01 x PIX-052B-06-01), F1(PIX-045B-27-02 X PIX-052B-06-01), F1(PIX045B-32-03 x PIX-052B-06-01), F1(PIX-044B-13-01 x MYR-29-09-05), F1(PIX-045B-27-02 x MYR-29-09-05), F1(PIX-045B-32-03 x MYR-29-09-05), and F1(PIX-052B-06-01 x MYR-29-0905)] had both the constituent parental lines with maximum levels of resistance to PepYMV (Table 2). These hybrids showed levels of resistance comparable to those of their constituent lines, as well as to that of the resistant control Criollo de Morellos-334 (asymptomatic plants from 88.9 to $100 \%$ ). In contrast, in the hybrids produced from one parent highly resistant to PepYMV and one susceptible parent, the percentage of asymptomatic plants ranged from 74.9 to $100 \%$, and in four of these cases, this percentage was significantly less than that obtained for Criollo de Morellos-334 and for the respective resistant parental line. These results suggest that the allele(s) that control(s) resistance to PepYMV has(have) incomplete dominance, and that slightly greater levels of resistance may be obtained in hybrids when both the parents are resistant. In a similar study of assessment of resistance to PepYMV, Nascimento et al. (2007) obtained results similar to those observed here for some genotypes assessed. The lowest level of resistance found in the experimental hybrids was that of the hybrid F1(PIX-044B-01-01 x Charleston Belle) (61.9\% asymptomatic plants), in which the resistant parent itself (PIX-044B-01-01) showed intermediate levels of resistance $(78.3 \%$ asymptomatic plants), a reflection of line PIX-044B-01-01 possibly not being homozygous for the trait in question.

Of the 30 experimental hybrids tested in regard to reaction to $M$. incognita, twenty were obtained from at least one resistant parent: Carolina
Wonder, Charleston Belle or Myr-2909-05, and they had reproduction indices $(\leq 6.2 \%)$ and reproduction factors $(\leq 1.8)$ that did not differ from those of the respective resistant lines nor from that of the control Criollo de Morelos-334, but they were significantly less than those of the susceptible control Magnata Super (Table 3). The commercial hybrids (Konan-R, Magali-R, Marta-R, Stephany and Mallorca), as well as 10 other experimental hybrids in which both the parental lines were susceptible, like the control Magnata Super, also proved to be susceptible to the nematode, with reproduction indices $\geq 136 \%$ and reproduction factors $\geq 29$.9. The amplitudes of variation of the reproduction indices (RI\%) and of the reproduction factors (RF) among the resistant lines was $0.2 \leq \mathrm{RI} \% \leq 2.7$ and $0.0 \leq \mathrm{RF} \leq 0.3$, amplitudes similar to those found for the hybrids [F1(Carolina Wonder x MYR-29-09-05) and F1(Charleston Belle x MYR-29-09-05)], composed of two resistant parents. The hybrids in which only one of the parents is resistant to $M$. incognita showed slightly greater amplitudes of variation for $\mathrm{RI} \%$ and for $\mathrm{RF}, 0.1 \leq \mathrm{RI} \% \leq 6.2$ and $0.0 \leq \mathrm{RF} \leq 1.8$. An allelic dosage effect is evident; two alleles for resistance in the same hybrid confer a slightly higher level of resistance than when only one allele is present. In the case of allelism between the $N$ gene present in Carolina Wonder/Charleston Belle (Fery et al., 1998) and the resistance allele present in MYR-29-09-05, it may be concluded that the degree of dominance of the gene is incomplete, however very near 1 .

Five hybrids were identified with simultaneous resistance to $P$. capsici, PepYMV and M. incognita: F1(PIX044B-01-01 x Carolina Wonder), F1(PIX-044B-13-01 x Carolina Wonder), F1(PIM-013 x MYR-2909-05), F1(PIX-045B-32-03 x MYR29-09-05) and F1(PIX-052B-06-01 x MYR-29-09-05). From the perspective of varietal resistance to the three pathogens, the four last ones are ready to be introduced on the market since their levels of resistance for each one of the pathogens are comparable to those of the best resistant genotypes. The hybrid 
F1(PIX-044B-01-01 x Carolina Wonder) would require previous selection within the line PIX-044B-01-01 with a view toward homozygosity for resistance to PepYMV.

For all the pathogens, $P$. capsici, PepYMV and $M$. incognita, there was evidence that hybrids with two resistant parental lines show levels of resistance slightly greater than those with only one resistant parental line. The case of allelism between the various sources of resistance used for each pathogen may be explained by gene activity of incomplete dominance.

\section{ACKNOWLEDGEMENTS}

To the Fundação de Amparo à Pesquisa do Estado de Minas Gerais (FAPEMIG), to the Conselho Nacional de Desenvolvimento Científico e Tecnológico (CNPq), to the Coordenação de Aperfeiçoamento de Pessoal de Nível Superior (CAPES), to the Universidade Federal de Lavras (UFLA), and to the company HortiAgro Sementes S.A for their support.

\section{REFERENCES}

BONETTI, JIS; FERRAZ, S. 1981. Modificações do método de Hussey \& Barker para extração de ovos de Meloidogyne exígua em raízes de cafeeiro. Fitopatologia Brasileira 6: 553-561.

CANDOLE, BL; CONNER, PJ; JI, P. 2010.
Screening Capsicum annuum accessions for resistance to six isolates of Phytophthora capsici. HortScience 45: 254-259.

ECHER, MM; COSTA, CP. 2002. Reaction of sweet pepper to the potato virus $Y\left(\mathrm{PVY}^{\mathrm{m}}\right)$. Scientia Agricola 59: 309-314.

FAZARI, A; PALLOIX, A; WANG, L; HUA, MY; SAGE-PALLOIX, A; ZHANG, BX; DJIANCAPORALINO, C. 2012. The root-knot nematode resistance $N$-gene co-localizes in the $\mathrm{Me}$-genes cluster on the pepper (Capsicum annuиm L.) P9 chromosome. Plant Breeding 131: 665-673.

FERY, RL; DUKES, PD; THIES, JA. 1998. 'Carolina Wonder' and 'Charleston Belle': southern root-knot nematode - resistant bell peppers. HortScience 33: 900-902.

GOMIDE, ML; MALUF, WR; GOMES, LAA. 2008. Capacidade de combinação de linhagens de pimentão (Capsicum annuum L.). Ciência e Agrotecnologia 32: 740-748.

LOPES, CA; ÁVILA, AC. 2003. Doenças do pimentão: diagnose e controle. Brasília: Embrapa Hortaliças. 96p.

LUCINDA, N; ROCHA, WB; INOUE-NAGATA, AK; NAGATA, T. 2012. Complete genome sequence of pepper yellow mosaic virus, a potyvirus, occurring in Brazil. Archives of Virology 157: 1397-1401.

MCGREGOR, C; WATERS, V; NAMBEESAN, S; MACLEAN, D; CANDOLE, BL; CONNER, P. 2011. Genotypic and phenotypic variation among pepper accessions resistant to Phytophthora capsici. HortScience 46: 1235-1240.

MONROY-BARBOSA, A; BOSLAND, PW. 2008. Genetic analysis of Phytophthora root rot race-specific resistance in Chile pepper. Journal of the American Society for Horticultural Science 133: 825-829.

NASCIMENTO, IR; MALUF, WR; FARIA, MV; VALLE, LAC; MENEZES, CB; BENITES, FRG. 2004. Capacidade combinatória e ação gênica na expressão de caracteres de importância econômica em pimentão. Ciência e Agrotecnologia 28: 251-260.

NASCIMENTO, IR; MALUF, WR; GONÇALVES, LD; FARIA, MV; RESENDE, JTV; NOGUEIRA, DW. 2010. Capacidade combinatória de linhagens de pimentão a partir de análise dialélica multivariada. Acta Scientiarum 32: 235-240.

NASCIMENTO, IR; VALE, LAC; MALUF, WR; GONÇALVES, LD; GOMES, LAA; MORETO, P; LOPES, EAGL. 2007. Reação de híbridos, linhagens e progênies de pimentão à requeima causada por Phytophthora capsici e ao mosaico amarelo causado por Pepper yellow mosaic virus (PepYMV). Ciência e Agrotecnologia 31: 121-128.

NOGUEIRA, DW. 2010. Seleção assistida por marcadores moleculares e capacidade combinatória de linhagens de pimentão com resistência múltipla a doenças. Lavras: UFLA. 80p (Tese doutorado).

NOGUEIRA, DW; NOGUEIRA, DG; MALUF, WR; MACIEL, GM; FIGUEIRA, AR; MENEZES, CB. 2012. Seleção assistida com uso de marcador molecular para resistência a potyvírus em pimentão. Pesquisa Agropecuária Brasileira 47: 955-963.

THIES, JA; ARISS, JJ. 2009. Comparison between the $N$ and $\mathrm{Me} 3$ genes conferring resistance to the root-knot nematode (Meloidogyne incognita) in genetically different pepper lines (Capsicum annuum). European Journal of Plant Pathology 125: 545-550. 